\title{
Socioeconomic Factors and Use of Antibiotics by Dairy Farmers vis-a-vis Antibiotic Resistance
}

\author{
Vikash Kumar* and Jancy Gupta \\ Division of Dairy Extension, ICAR- National Dairy Research Institute, Karnal, Haryana, India \\ *Corresponding author
}

\section{A B S T R A C T}

\section{Keywords}

Antibiotic

conservation,

Judicious use, One-

health, Preventive,

Stakeholders,

Symptom-relief

Article Info

Accepted:

26 October 2017

Available Online:

10 January 2018
The use of antibiotics for preventive purpose and symptom relief are common in India. One third $(67 \%)$ of the global increase in antibiotic consumption in livestock animals over 2010-2030, will be evident in Low and Middle Income Countries like India (Van Boeckel et al., 2015). Reinforcing the education and excellence among stakeholders about antibiotic conservation practices, issue of antibiotic resistance and the One Health approach can play crucial role in leading the resistance from farm-to-fork (OIE, 2016). In the study, two districts namely Karnal and Kurukshetra were purposively selected from Eastern zone of Haryana state. In which, 4 villages from each district were selected randomly. From each village 21 farmers were randomly selected through random stratified sampling methods constituting the total respondents to 168.Mean, standard deviation, frequency and cumulative square root frequency were statistical tools to be used. The educational campaigns increase awareness about the resistance and reinforcing the education and fostering the excellence among stakeholders about antibiotic conservation practices is needed. The large farmers performed judicious use of antibiotics, higher incomes helped them to adopt better management practices at their farms. A wellstructured target segmentation strategy to percolate the policies for small, medium and large dairy farmers can be designed by considering the socio-economic conditions.

\section{Introduction}

The irrational use of antibiotics is prevalent among the dairy farmers. If it continues with same pace, then between 2010 and 2030, its global consumption in the livestock sector can increase by about $67 \%$. The substantial part of this increase in antibiotics consumption will be determined by a shift in farming techniques in major developing countries (Van Boeckel et al., 2015). In the world, only 48 countries $(25 \%$ of the 194 WHO member countries) considered national plans in to operation to tackle antibiotics resistance (Cecchini et al., 2015).
An updated preclinical paradigm is needed to assess the risk of resistance development in early preclinical development. This change is feasible and is not limited by methodology but rather by tradition (Sommer et al., 2017).

A paradigm shift is required in utilization of conventional antibiotics and its screening for next-generation to improve efficacy to treat biofilms associated with recurrent and device related infections in the development of antibiotics (Ceri et al., 2010). The constant advancement in molecular techniques and 
next-generation sequencing led to a paradigm shift in the thoughts of health dynamics (Falentin et al., 2016). In the United States of America, the use of antibiotics including antibiotics in livestock sector accounts for about $80 \%$ of the total consumption annually (FDA, 2010).

The resistance crossed the boundary of countries, e.g. carbapenem resistant strain of Klebsiella identified in 2003 needed only 5 years to spread from the United States, where it was, to Israel (2005) to the United Kingdom, Italy and Colombia (2008) (McKenna, 2013).

Developing countries (OECD) may face cumulative losses for USD 2.9 trillion (about $0.16 \%$ of their GDP) by 2050 as compared to world (Cecchini et al., 2015). One third $(67 \%)$ of the global increase in antibiotic consumption in livestock animals over 20102030, will be evident in Low and Middle Income Countries like India (Van Boeckel et al., 2015). National guidelines for antibiotic use were introduced and implemented in India since 2013 (Founou et al., 2016).

One health approach is multifaceted, comprehensive and integrated processes imperative to ensure food safety, combat infectious diseases, preserve the efficacy of antibiotics for future generations and control the spread of antibiotic resistance (Founou et al., 2016).

The One Health science seems to fall short when it comes to understanding the economic forces behind many emerging infectious diseases; there is a further gap to be bridged between the biomedical, environmental and animal sciences, and the social sciences (Robinson et al., 2016). This approach endorses the supranational entities such as WHO, FAO, and OIE referred as the "Tripartite Alliance (WHO, 2015).
Interconnected interests of stakeholders integrating human health, veterinary medicine and the environment, surveillance should be established.

Reinforcing the education and excellence among veterinarians about antibiotic conservation practices, issue of antibiotic resistance and the One Health approach can play crucial role in leading the resistance from farm-to-fork (OIE, 2016). WHO Global Action Plan and FAO Action Plan advocated multifaceted strategies with One Health approach, are urgently required to prevent the transmission of resistance and infectious diseases from farm-to-fork and preserve the efficacy of antibiotics to promote food security resulting global health (Founou et al., 2016).

There is need to formulate the strategies regarding the prudent use of antibiotics among the Indian farmers. Their socioeconomic conditions need to be addressed to design the strategies for the small, medium and large dairy farmers. Considering above view points, the present study was conducted to address the issue of antibiotic resistance.

\section{Materials and Methods}

In the study, two districts namely Karnal and Kurukshetra were purposively selected from Eastern zone of Haryana state. In which, 4 villages from each district were selected randomly. From each village 21 farmers were randomly selected through random stratified sampling methods.

The farmers were categorised into small, medium and large farmers on the basis of their herd size, constituting a total of 168 farmers as respondents. Mean, standard deviation, frequency and cumulative square root frequency were statistical tools to be used. 


\section{Results and Discussion}

\section{Socio-economic conditions of dairy farmers}

Age

Age determines the maturity of the individual which is guided by experience and exposure to the situation for the person. Result indicated that half of respondents $(52.38 \%)$ were in the middle category of age, followed by $25 \%$ of the respondents in the category of old age and the remaining belonged to the category of young age (Table 1).

\section{Family size}

Majority (59.52\%) of respondents belonged to medium family size comprising of 5 to 10 members, followed by $28 \%$ of respondents in low family size category. Family size influenced the annual income, labour availability and per capita milk consumption (Table 2).

\section{Family type}

Majority of respondents (76.19\%) belonged to joint family and remaining respondents belong to nuclear family. Joint family has the advantage of availability of surplus labour for the agriculture and allied activities and proportion of joint family is high as compared to nuclear family in the rural society (Table $3)$.

\section{Education}

Education broadens the mental horizon at the same time it increases the readiness and predisposition to the acquisition of the new and novel ideas. Majority of small farmers were illiterate followed by functionally literate and $21.78 \%$ had middle level of education. Among medium farmers $29.31 \%$ were literate up to middle level followed by $22.41 \%$ farmers having primary level and
$14.66 \%$ of farmers were educated up to secondary level of education. Among large farmers, the education level was highest for middle level followed by secondary level of education $(24.19 \%)$ which was higher as compared to small and medium farmers. Thus, education level varied from small farmers to medium farmers and was found highest among large farmers thus showing a wide variation from illiterate to the graduate level of education.

\section{Social participation}

Majority of small farmers $(40.51 \%)$ were reported to have medium level of social participation and $33.86 \%$ of them were found to have low level of social participation followed by $25.63 \%$ having high level social participation. Majority of medium farmers $(39.66 \%)$ were found to have medium level of social participation and $33.03 \%$ of them had low level of social participation followed by $27.31 \%$ having high level social participation (Table 5).

Majority of large farmers (42.07\%) were having medium level of social participation and $29.14 \%$ of them had low level of social participation followed by $28.80 \%$ having high level social participation.

\section{Land holding}

Majority of the small holder dairy farmers $(40.69 \%)$ were having land holding of less than 1 hectare, followed by $31 \%$ of farmers having land holding more than 2 hectare. The land holding was higher among medium dairy farmers as compared to small dairy farmers, in which majority (49.24\%) of them belonged to land holding 2.25 to 4.75 hectare followed by $27.07 \%$ of farmers having land holding more than 4.45 hectare (Table 6). The size of land holding was highest among large dairy farmers as compares to small and medium dairy farmers. Out of which, $50 \%$ of large 
dairy farmers were having the land holding of 3.50 to 7 hectare followed by $27.38 \%$ of farmers were having the land holding more than 7 hectare.

\section{Herd size}

Majority of small holder dairy farmers (76\%) had the average herd size (3 Livestock Animal Unit). Majority of medium dairy farmers $(71 \%)$ had the average herd size of 7 animals belonged to the middle category. Among large dairy farmers, majority $(75 \%)$ of them had the average herd size of 13 Livestock Animal Unit (Table 7).

Milk production, milk consumption and milk sale

Majority of the small farmers (51.19\%) belonged to the medium level of milk production (5-11 lits./day) followed by $25.01 \%$ of them in low and $23.80 \%$ in high category. It was revealed that $77 \%$ of the respondents consumed 2.23 to 5.2 litres of milk for household purpose (Table 8). It was found that $14.28 \%$ of the respondents were consuming less than 2.3 litres of milk per day belonged to low category of milk consumption.

It was revealed that $22.62 \%$ of the respondents did not sell the milk thus consumed the whole milk at household level. About $27.38 \%$ of the respondents sold 3.5 to 8.2 litres of milk which belonged to the medium category of the classification. Majority of medium farmers $(48.81 \%)$ belonged to the medium level of milk production (9-16 lits. / day) followed by 28.57 $\%$ who belonged to low category of milk production ( $<9$ lits./ day) and remaining 22.62 $\%$ of respondents belonged to high category. It was revealed that $75.38 \%$ of the respondents consumed 4.5 to 9.2 litres of milk for household purpose. It was found that $14.29 \%$ of the respondents consumed less than 4.5 litres of milk per day belonged to low category of milk consumption. It was found that $59.52 \%$ of the respondents sold the milk belonged to the middle category (7.5-13.2 lits./day) of milk sale. About $25.6 \%$ of the respondents sold more than 13.2 litres of milk that belonged to the high category of the classification.

Majority of the respondents (42.26\%) belonged to the medium level of milk production (16-26 lits./day) followed by 33.93 $\%$ of the respondents belonged to low category of milk production ( $<16$ lits./day) and remaining $23.81 \%$ of respondents were in high category. It was revealed that $77.38 \%$ of the respondents consumed 7.5 to 9.2 litres of milk for household purpose (Table 9).It was found that $14.28 \%$ of the respondents consumed less than 7.5 litres of milk per day belonged to low category of milk consumption. It was found that $52.98 \%$ of the respondents sold the milk belonged to the middle category (13.5-20.4 lits./ day) of milk sale. About $25 \%$ of the respondents sold more than 20.4 litres of milk belonged to the high category of the classification.

\section{Annual Income}

Majority of the small farmers $(73.7 \%)$ belonged to the medium level of income of Rs. 91678-Rs. 242454 per annum, while, $15.17 \%$ of the them were from high income level, earning more than Rs. 122638 per annum and $11.8 \%$ of them were earning less than Rs. 51660 per annum. Majority of the medium farmers $(63.12 \%)$ belonged to the medium level of income of Rs. 91678- Rs. 242454 per annum, while, $21.61 \%$ of the them were from high income level, earning more than Rs. 242454 per annum and 15.27 $\%$ of them were earning less than Rs. 91678 per annum. Among large farmers, majority of them $(70.54 \%)$ belonged to the medium level of income of Rs. 203647- Rs. 328452 per annum. 
Table.1 Distribution of respondents as per age $(n=168)$

\begin{tabular}{|l|c|c|c|}
\hline Category & Karnal & Kurukshetra & Total \\
\hline Young Age (<35) & 25 & 20.24 & 22.62 \\
\hline Middle Age (35-50) & 48.81 & 55.95 & 52.38 \\
\hline Old age (>50) & 26.19 & 23.81 & 25.00 \\
\hline
\end{tabular}

(Numerical figures indicate \%)

Table.2 Distribution of respondents as per family size $(n=168)$

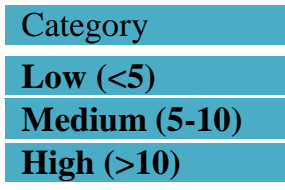

\begin{tabular}{|c|}
\hline Karnal \\
\hline 25 \\
\hline 55.95 \\
\hline 19.05 \\
\hline
\end{tabular}

\begin{tabular}{|c|}
\hline Kurukshetra \\
\hline 30.95 \\
\hline 63.10 \\
\hline 5.95 \\
\hline
\end{tabular}

\begin{tabular}{|c|}
\hline Total \\
\hline 27.98 \\
\hline 59.52 \\
\hline 12.5 \\
\hline
\end{tabular}

(Numerical figures indicate \%)

Table.3 Distribution of respondents as per family type $(n=168)$

\begin{tabular}{|l|l|}
\hline Family type \\
\hline Joint \\
\hline Nuclear
\end{tabular}

\begin{tabular}{|c|}
\hline Karnal \\
\hline 67.86 \\
\hline 32.14 \\
\hline
\end{tabular}

\begin{tabular}{|c|}
\hline Kurukshetra \\
\hline 84.52 \\
15.48 \\
\hline
\end{tabular}

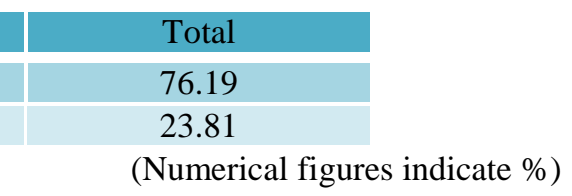

Table.4 Distribution of respondents as per education $(n=168)$

\begin{tabular}{|l|}
\hline Educational level \\
\hline No schooling \\
\hline Functionally literate \\
\hline Primary \\
\hline Middle \\
\hline Secondary \\
\hline Higher secondary \\
\hline Graduate \\
\hline \\
\hline Educational level \\
\hline No schooling \\
\hline Functionally literate \\
\hline Primary \\
\hline Middle \\
\hline Secondary \\
\hline Higher secondary \\
\hline Graduate \\
\hline \\
\hline Educational level \\
\hline No schooling \\
\hline Functionally literate \\
\hline primary \\
\hline Middle \\
\hline Secondary \\
\hline Higher secondary \\
\hline Graduate \\
\hline
\end{tabular}

\begin{tabular}{|c|}
\hline Karn \\
\hline 25.8 \\
\hline 29.31 \\
\hline 5.17 \\
\hline 22.41 \\
\hline 6.90 \\
\hline 6.90 \\
\hline 3.45 \\
\hline
\end{tabular}

\section{Small farmers $(n=56)$}

\begin{tabular}{|c|}
\hline Karna \\
\hline 13.79 \\
\hline 5.17 \\
\hline 25.86 \\
\hline 32.76 \\
\hline 15.52 \\
\hline 5.17 \\
\hline 1.72 \\
\hline
\end{tabular}

Medium farmers $(\mathrm{n}=\mathbf{5 6})$

\begin{tabular}{|c|c|}
\hline Kurukshetra & Total \\
\hline 27.59 & 26.72 \\
\hline 18.97 & 24.14 \\
\hline 8.62 & 6.90 \\
\hline 21.14 & 21.78 \\
\hline 8.62 & 7.76 \\
\hline 8.17 & 7.53 \\
\hline 6.90 & 5.17 \\
\hline
\end{tabular}

\begin{tabular}{|c|c|c|}
\hline \multicolumn{2}{|c|}{ Large farmers $(\mathbf{n = 5 6})$} & \\
\hline Karnal & Kurukshetra & Total \\
\hline 12.50 & 16.67 & 14.58 \\
\hline 5.36 & 9.26 & 7.31 \\
\hline 16.07 & 18.52 & 17.29 \\
\hline 26.79 & 24.07 & 25.43 \\
\hline 28.00 & 20.37 & 24.19 \\
\hline 5.93 & 7.41 & 6.67 \\
\hline 5.36 & 3.70 & 4.53 \\
\hline & & (Numerical figures indicate \%) \\
\hline
\end{tabular}


Table.5 Distribution of respondents as per social participation $(n=168)$

\begin{tabular}{|c|c|c|c|}
\hline \multicolumn{4}{|c|}{ Small farmers $(n=56)$} \\
\hline Category & Karnal & Kurukshetra & Total \\
\hline $\operatorname{Low}(<2.4)$ & 31.31 & 36.42 & 33.86 \\
\hline Medium (2.4-4.7) & 41.79 & 39.24 & 40.51 \\
\hline High $(>4.7)$ & 26.90 & 24.34 & 25.63 \\
\hline \multicolumn{4}{|c|}{ Medium farmers $(n=56)$} \\
\hline Category & Karnal & Kurukshetra & Total \\
\hline Low $(<3.8)$ & 32.07 & 33.98 & 33.03 \\
\hline Medium (3.8-6.2) & 39.02 & 40.30 & 39.66 \\
\hline High $(>6.2)$ & 28.91 & 25.72 & 27.31 \\
\hline \multicolumn{4}{|c|}{ Large farmers $(n=56)$} \\
\hline Category & Karnal & Kurukshetra & Total \\
\hline Low $(<4.1)$ & 30.45 & 27.83 & 29.14 \\
\hline Medium (4.1-7.6) & 41.03 & 43.10 & 42.07 \\
\hline High $(>7.6)$ & 28.52 & 29.07 & 28.80 \\
\hline
\end{tabular}

Table.6 Distribution of respondents as per land holding $(\mathrm{n}=168)$

\begin{tabular}{|c|c|c|c|}
\hline \multicolumn{4}{|c|}{ Small holder dairy Farmers $(n=56)$} \\
\hline Category & Karnal & Kurukshetra & Total \\
\hline$(<\mathbf{H a})$ & 41.67 & 39.71 & 40.69 \\
\hline (1-2 Нa) & 30.85 & 25.81 & 28.33 \\
\hline$(>2 \mathrm{Ha})$ & 27.38 & 34.5 & 30.94 \\
\hline \multicolumn{4}{|c|}{ Medium Farmers $(n=56)$} \\
\hline Category & Karnal & Kurukshetra & Total \\
\hline$(<2.25 \mathrm{Ha})$ & 23.82 & 24.38 & 24.10 \\
\hline$(2.25-4.75 H a)$ & 45.33 & 53.14 & 49.24 \\
\hline$(>4.45 \mathrm{Ha})$ & 30.95 & 23.18 & 27.07 \\
\hline \multicolumn{4}{|c|}{ Large Dairy Farmers $(n=56)$} \\
\hline Category & Karnal & Kurukshetra & Total \\
\hline$(<3.50 \mathrm{Ha})$ & 23.81 & 21.43 & 22.62 \\
\hline (3.50-7Ha) & 47.62 & 52.38 & 50 \\
\hline$(>7 \mathrm{Ha})$ & 28.57 & 26.19 & 27.38 \\
\hline
\end{tabular}

(Numerical figures indicate \%)

Table.7 Distribution of respondents as per herd size $(n=168)$

\begin{tabular}{|c|c|c|c|}
\hline \multicolumn{4}{|c|}{ Small farmer $(n=56)$} \\
\hline Average herd size & Karnal & Kurukshetra & Total \\
\hline 3 SAU & 74 & 78 & 76 \\
\hline \multicolumn{4}{|c|}{ Medium farmer $(n=56)$} \\
\hline Average herd size & Karnal & Kurukshetra & Total \\
\hline $7 \mathrm{SAU}$ & 69 & 73 & 71 \\
\hline \multicolumn{4}{|c|}{ Large farmers $(n=56)$} \\
\hline Average herd size & Karnal & Kurukshetra & Total \\
\hline $13 \mathrm{SAU}$ & 76 & 74 & 75 \\
\hline
\end{tabular}


Table.8 Distribution of small farmers as per milk production, milk consumption and milk sale $(n=56)$

\begin{tabular}{|c|c|c|c|}
\hline \multicolumn{4}{|c|}{ Milk production per day (Litres) } \\
\hline Category & Karnal & Kurukshetra & Total \\
\hline low (<5 Lit/ day) & 21.43 & 28.58 & 25.01 \\
\hline medium (5-11 lit/ day) & 53.57 & 48.81 & 51.19 \\
\hline high (>11 lit/ day) & 25 & 22.61 & 23.80 \\
\hline \multicolumn{4}{|c|}{ Milk consumption per day (Litres) } \\
\hline Category & Karnal & Kurukshetra & Total \\
\hline Low $(<2.3)$ & 20.24 & 8.33 & 14.28 \\
\hline Medium (2.3-5.2) & 70.24 & 84.52 & 77.38 \\
\hline High $(>5.2)$ & 9.52 & 7.15 & 8.335 \\
\hline \multicolumn{4}{|c|}{ Milk sale per day (Litres) } \\
\hline Category & Karnal & Kurukshetra & Total \\
\hline No sale & 16.66 & 28.57 & 22.62 \\
\hline Low $(<3.5)$ & 13.09 & 13.1 & 13.09 \\
\hline Medium (3.5-8.2) & 59.54 & 46.42 & 27.38 \\
\hline High $(>8.2)$ & 10.71 & 11.91 & 11.31 \\
\hline
\end{tabular}

Table.9 Distribution of medium farmers as per milk production, milk consumption and milk sale $(n=56)$

\begin{tabular}{|l|c|c|c|c|}
\hline \multicolumn{4}{|c|}{ Milk production per day (Litres) } \\
\hline Category & Karnal & Kurukshetra & Total \\
\hline low (<9 Lit/ day) & 25 & 32.14 & 28.57 \\
\hline medium (9-16 lit/ day) & 47.62 & 50 & 48.81 \\
\hline high (>16 lit/ day) & 27.38 & 17.86 & 22.62 \\
\hline & Milk consumption per day (Litres) & \\
\hline Category & Karnal & Kurukshetra & Total \\
\hline Low (<4.5) & 20.24 & 8.33 & 14.29 \\
\hline Medium (4.5-9.2) & 70.24 & 83.52 & 75.38 \\
\hline High (>9.2) & 9.52 & 10.52 & 18.67 \\
\hline & Milk Sale per day (Litres) & \\
\hline Category & Karnal & Kurukshetra & Total \\
\hline Low (<7.5) & 13.1 & 16.67 & 14.88 \\
\hline Medium (7.5-13.2) & 66.67 & 52.38 & 59.52 \\
\hline High (>13.2) & 20.24 & 30.95 & 25.6 \\
\hline
\end{tabular}


Table.10 Distribution of large farmers as per milk production, milk consumption and milk sale $(n=56)$

\begin{tabular}{|l|c|c|c|c|}
\hline \multicolumn{4}{c}{ Milk production per day (Litres) } \\
\hline Category & Karnal & Kurukshetra & Total \\
\hline low (<16Lit/ day) & 32.14 & 35.71 & 33.93 \\
\hline medium (16-26 lit/ day) & 45.24 & 39.29 & 42.26 \\
\hline high (>26 lit/ day) & Milk consumption per day (Litres) & 23.81 \\
\hline & & Karnal & Kurukshetra & Total \\
\hline Category & 22.62 & 8.33 & 14.285 \\
\hline Low (<7.5) & & 70.24 & 84.52 & 77.38 \\
\hline Medium (7.5-9.2) & 9.52 & 7.14 & 8.33 \\
\hline High (>9.2) & Milk sale per day (Litres) & Total \\
\hline Category & Karnal & Kurukshetra & 22.02 \\
\hline Low (<13.25) & 23.81 & 20.24 & 52.98 \\
\hline Medium (13.5-20.4) & 50 & 55.95 & 25 \\
\hline High (>20.4) & & & 23.81 & (Numerical figures indicate \%) \\
\hline
\end{tabular}

Table.11 Distribution of respondents as per annual income (Rs.) ( $\mathrm{n}=168)$

\begin{tabular}{|l|c|c|c|c|}
\hline & \multicolumn{2}{|c|}{ Small farmers $(\mathbf{n = 5 6})$} & \\
\hline Category & Karnal & Kurukshetra & Total \\
\hline Low $(<\mathbf{5 1 6 6 0 )}$ & 8.03 & 14.33 & 11.18 \\
\hline Medium (51660-122638) & 77.07 & 70.33 & 73.7 \\
\hline High (>122638) & 15 & 15.34 & 15.17 \\
\hline & Medium farmers $(\mathbf{n = 5 6 )}$ & \\
\hline Category & Karnal & Kurukshetra & Total \\
\hline Low (<91678) & 13.2 & 17.34 & 15.27 \\
\hline Medium (91678-242454) & 64.7 & 61.54 & 63.12 \\
\hline High (>242454) & 22.1 & 21.12 & 21.61 \\
\hline & Large farmers (n=56) & \\
\hline Category & Karnal & Kurukshetra & Total \\
\hline Low (<203647) & 16.83 & 15.83 & 16.33 \\
\hline Medium (203647-328452) & 69.52 & 71.57 & 70.54 \\
\hline High(>328374) & 13.65 & 12.6 & 13.13 \\
\hline
\end{tabular}


Table.12 Distribution of respondents according to mass media exposure $(n=168)$

\begin{tabular}{|l|c|c|c|c|}
\hline \multicolumn{5}{|c|}{ Small farmers $(\mathbf{n = 5 6})$} \\
\hline Category & Karnal & Kurukshetra & Total \\
\hline Low (<4) & 18.63 & 15.2 & 16.915 \\
\hline Medium (4-7) & 65.47 & 63.58 & 64.525 \\
\hline High (>7) & 15.9 & 21.22 & 18.56 \\
\hline & Medium farmers $(\mathbf{n = 5 6 )}$ & \\
\hline Category & Karnal & Kurukshetra & Total \\
\hline Low (<5) & 21.36 & 24.63 & 22.995 \\
\hline Medium (5-8) & 61.32 & 63.89 & 62.605 \\
\hline High (>8) & 17.32 & 11.48 & 14.4 \\
\hline & Large farmers (n=56) & \\
\hline Category & Karnal & Kurukshetra & Total \\
\hline Low (<7) & 12.45 & 14.37 & 13.41 \\
\hline Medium (7-10) & 63.46 & 61.62 & 62.54 \\
\hline High (>10) & 24.09 & 24.1 & 24.095 \\
\hline & & & (Numerical figures indicate \%) \\
\hline
\end{tabular}

Table.13 Distribution of respondents as per extension contact $(n=168)$

\begin{tabular}{|l|c|c|c|c|}
\hline \multicolumn{4}{|c}{ Small farmers $(\mathbf{n}=\mathbf{5 6})$} \\
\hline Category & Karnal & Kurukshetra & Total \\
\hline Low $(<\mathbf{3 )}$ & 11.90 & 22.62 & 17.26 \\
\hline Medium (3-7) & 75.00 & 73.81 & 74.40 \\
\hline High (>8) & 13.10 & 3.57 & 8.33 \\
\hline & Medium farmers $(\mathbf{n}=\mathbf{5 6})$ & \\
\hline Category & Karnal & Kurukshetra & Total \\
\hline Low (<5) & 40.48 & 42.86 & 41.67 \\
\hline Medium (5-10) & 48.81 & 52.38 & 50.60 \\
\hline High (>10) & 10.71 & 4.76 & 7.74 \\
\hline & Large farmers (n=56) & \\
\hline Category & Karnal & Kurukshetra & Total \\
\hline Low (<7) & 26.19 & 22.62 & 24.40 \\
\hline Medium (7-12) & 57.14 & 75.00 & 66.07 \\
\hline High (>12) & 16.67 & 2.38 & 9.52 \\
\hline & & & (Numerical figures indicate \%) \\
\hline
\end{tabular}


Table.14 Classification of respondents according to the factors considered while buying the antibiotics from medical store and feed store

\begin{tabular}{|c|c|c|c|c|}
\hline Sr. No. & Factors & Never (0) & Sometimes (1) & Always (2) \\
\hline \multicolumn{5}{|c|}{ Small farmers } \\
\hline 1. & Price as order of importance & 10.50 & 14.50 & 75.00 \\
\hline 2. & Brand Packaging as suggested by veterinary medicine store & 7.15 & 21.42 & 71.43 \\
\hline 3. & Quality in terms of previous experience & 25.00 & 23.21 & 51.79 \\
\hline 4. & Easy to obtain & 19.64 & 23.21 & 57.15 \\
\hline 5. & $\begin{array}{l}\text { Previous recommendation slip of veterinarian or the residual scanty } \\
\text { antibiotics brought by milk vender }\end{array}$ & 16.07 & 26.79 & 57.14 \\
\hline \multicolumn{5}{|c|}{ Medium farmers } \\
\hline 6. & Price as order of importance & 23.21 & 37.50 & 39.29 \\
\hline 7. & Brand Packaging as suggested by veterinary medicine store & 28.57 & 7.14 & 64.29 \\
\hline 8. & Quality in terms of previous experience & 21.42 & 10.71 & 67.87 \\
\hline 9. & Easy to obtain & 39.28 & 26.78 & 33.94 \\
\hline 10. & $\begin{array}{l}\text { Previous recommendation slip of veterinarian or the residual scanty } \\
\text { antibiotics brought by milk vender }\end{array}$ & 66.07 & 21.43 & 12.50 \\
\hline \multicolumn{5}{|c|}{ Large farmers } \\
\hline 11. & Price as order of importance & 67.86 & 19.64 & 12.50 \\
\hline 12. & Brand Packaging as suggested by veterinary medicine store & 7.14 & 8.93 & 83.93 \\
\hline 13. & Quality in terms of previous experience & 75.00 & 14.29 & 10.71 \\
\hline 14. & Easy to obtain & 66.07 & 17.86 & 16.07 \\
\hline 15. & $\begin{array}{l}\text { Previous recommendation slip of veterinarian or the residual scanty } \\
\text { antibiotics brought by milk vender }\end{array}$ & 89.29 & 7.14 & 3.57 \\
\hline
\end{tabular}

(Numerical figures indicate \%)

Table.15 Classification of respondents according to practice(s) perform/ focus while using the antibiotics

\begin{tabular}{|c|c|c|c|c|}
\hline Sr. No. & Practice(s) & Never $(0)$ & Sometimes (1) & Always (2) \\
\hline \multicolumn{5}{|c|}{ Small farmers $(n=56)$} \\
\hline 1. & $\begin{array}{l}\text { I always follow best practice when making decisions about, and } \\
\text { administering, antibiotics to my animals }\end{array}$ & 60.79 & 17.63 & 21.58 \\
\hline 2. & Every use of antibiotics on my animals is fully recorded & 70.67 & 16.95 & 12.38 \\
\hline 3. & Antibiotics are expensive and I minimize usage to reduce costs & 13.64 & 21.54 & 67.82 \\
\hline 4. & $\begin{array}{l}\text { Preventative use of antibiotics in the dairy herd helps me meet production } \\
\text { goals }\end{array}$ & 95.57 & 1.64 & 2.79 \\
\hline 5. & I always consult my vet before using antibiotics to treat an animal & 33.63 & 34.74 & 31.63 \\
\hline \multicolumn{5}{|c|}{ Medium farmers $(\mathrm{n}=56)$} \\
\hline 1. & $\begin{array}{l}\text { I always follow best practice when making decisions about, and } \\
\text { administering, antibiotics to my animals }\end{array}$ & 30.83 & 25.53 & 43.64 \\
\hline 2. & Every use of antibiotics on my animals is fully recorded & 30.66 & 32.82 & 36.52 \\
\hline 3. & Antibiotics are expensive and I minimize usage to reduce costs & 40.42 & 16.96 & 42.62 \\
\hline 4. & $\begin{array}{l}\text { Preventative use of antibiotics in the dairy herd helps me meet production } \\
\text { goals }\end{array}$ & 56.63 & 28.62 & 14.75 \\
\hline 5. & I always consult my vet before using antibiotics to treat an animal & 9.35 & 27.92 & 62.73 \\
\hline \multicolumn{5}{|c|}{ Large farmers $(n=56)$} \\
\hline 1. & $\begin{array}{l}\text { I always follow best practice when making decisions about, and } \\
\text { administering, antibiotics to my animals }\end{array}$ & 13.73 & 13.52 & 72.75 \\
\hline 2. & Every use of antibiotics on my animals is fully recorded & 9.84 & 21.38 & 68.78 \\
\hline 3. & Antibiotics are expensive and I minimize usage to reduce costs & 18.80 & 26.94 & 54.26 \\
\hline 4. & $\begin{array}{l}\text { Preventative use of antibiotics in the dairy herd helps me meet production } \\
\text { goals }\end{array}$ & 19.25 & 31.81 & 48.94 \\
\hline 5. & I always consult my vet before using antibiotics to treat an animal & 2.08 & 12.92 & 85.00 \\
\hline
\end{tabular}

(Numerical figures indicate \%) 
Table.16 The classification of respondents according to responses and the prevailing practices which are accelerating factor as well as a matter of awareness about antibiotic resistance

\begin{tabular}{|c|c|c|c|c|}
\hline Sr. No & Practice(s) & Never & Sometime & Always \\
\hline \multicolumn{5}{|c|}{ Small farmers $(n=56)$} \\
\hline 1 . & $\begin{array}{l}\text { Do you discriminate usage of antibiotics for lactating and non- } \\
\text { lactating cattle, calf and milch animal? }\end{array}$ & 24.64 & 18.51 & 56.85 \\
\hline 2. & Do you give the recommended dose of without any alteration? & 16.75 & 39.83 & 43.42 \\
\hline 3. & $\begin{array}{l}\text { Do you sale the milk and it's product during the administration of } \\
\text { antibiotics? }\end{array}$ & 2.04 & 0.00 & 97.96 \\
\hline 4. & $\begin{array}{l}\text { Do you discard the use of milk of treated animals for the domestic } \\
\text { consumption? }\end{array}$ & 96.53 & 2.04 & 1.43 \\
\hline 5. & $\begin{array}{l}\text { Whether you demand for prescribing antibiotics to the } \\
\text { veterinarians exclusively? }\end{array}$ & 24.53 & 18.94 & 56.53 \\
\hline 6. & $\begin{array}{l}\text { Do you complete the course of prescription even when symptoms } \\
\text { of disease disappear? }\end{array}$ & 68.96 & 27.63 & 3.41 \\
\hline 7. & $\begin{array}{l}\text { Do you use the left over antibiotics for the common disease(s) } \\
\text { which appears regularly? }\end{array}$ & 14.75 & 6.83 & 78.42 \\
\hline 8. & Do you share antibiotics with your neighbour/ relatives? & 23.53 & 29.94 & 46.53 \\
\hline \multicolumn{5}{|c|}{ Medium farmers $(\mathrm{n}=56)$} \\
\hline 9 . & $\begin{array}{l}\text { Do you discriminate usage of antibiotics for lactating and non- } \\
\text { lactating cattle, calf and milch animal? }\end{array}$ & 46.85 & 20.52 & 32.63 \\
\hline 10. & Do you give the recommended dose of without any alteration? & 26.65 & 5.71 & 67.64 \\
\hline 11. & $\begin{array}{l}\text { Do you sale the milk and it's product during the administration of } \\
\text { antibiotics? }\end{array}$ & 4.38 & 1.98 & 93.64 \\
\hline 12. & $\begin{array}{l}\text { Do you discard the use of milk of treated animals for the domestic } \\
\text { consumption? }\end{array}$ & 95.63 & 4.53 & 0.00 \\
\hline 13. & $\begin{array}{l}\text { Whether you demand for prescribing antibiotics to the } \\
\text { veterinarians exclusively? }\end{array}$ & 34.64 & 38.69 & 26.67 \\
\hline 14. & $\begin{array}{l}\text { Do you complete the course of prescription even when symptoms } \\
\text { of disease disappear? }\end{array}$ & 78.42 & 11.57 & 10.01 \\
\hline 15. & $\begin{array}{l}\text { Do you use the left over antibiotics for the common disease(s) } \\
\text { which appears regularly? }\end{array}$ & 11.74 & 1.73 & 86.53 \\
\hline 16. & Do you share antibiotics with your neighbour/ relatives? & 91.74 & 5.97 & 2.29 \\
\hline \multicolumn{5}{|c|}{ Large farmers $(n=56)$} \\
\hline 17. & $\begin{array}{l}\text { Do you discriminate usage of antibiotics for lactating and non- } \\
\text { lactating cattle, calf and milch animal? }\end{array}$ & 45.74 & 40.62 & 13.64 \\
\hline 18. & Do you give the recommended dose of without any alteration? & 11.64 & 5.04 & 83.32 \\
\hline 19. & $\begin{array}{l}\text { Do you sale the milk and it's product during the administration of } \\
\text { antibiotics? }\end{array}$ & 3.85 & 3.62 & 92.53 \\
\hline 20. & $\begin{array}{l}\text { Do you discard the use of milk of treated animals for the domestic } \\
\text { consumption? }\end{array}$ & 91.75 & 4.64 & 3.61 \\
\hline 21. & $\begin{array}{l}\text { Whether you demand for prescribing antibiotics to the } \\
\text { veterinarians exclusively? }\end{array}$ & 68.40 & 18.86 & 12.74 \\
\hline 22. & $\begin{array}{l}\text { Do you complete the course of prescription even when symptoms } \\
\text { of disease disappear? }\end{array}$ & 87.96 & 2.74 & 9.3 \\
\hline 23. & $\begin{array}{l}\text { Do you use the left over antibiotics for the common disease(s) } \\
\text { which appears regularly? }\end{array}$ & 2.01 & 6.42 & 91.57 \\
\hline 24. & Do you share antibiotics with your neighbour/ relatives? & 96.12 & 1.35 & 2.53 \\
\hline
\end{tabular}


(Numerical figures indicate \%)

Table.17 Resistance in bacteria and resistant antibiotics as observed by veterinarians

\begin{tabular}{|l|l|c|}
\hline Sr. No. & Bacteria & Resistance \\
\hline $\mathbf{1}$ & Coliform & 17.39 \\
\hline $\mathbf{2}$ & Pseudomonas aeruginosa & 39.13 \\
\hline $\mathbf{3}$ & Staphylococcus aureus & 30.43 \\
\hline $\mathbf{4}$ & Streptococcus & 34.78 \\
\hline $\mathbf{5}$ & Escherichia coli & 52.17 \\
\hline $\mathbf{6}$ & Klebsiella & 13.04 \\
\hline $\mathbf{7}$ & Salmonella & 26.09 \\
\hline Sr. No. & Antibiotics & Resistance \\
\hline $\mathbf{1}$ & Gentamycin & 65.22 \\
\hline $\mathbf{2}$ & Penicillin & 72.34 \\
\hline $\mathbf{3}$ & Tetracycline & 21.77 \\
\hline $\mathbf{4}$ & Beta-lactams & 34.45 \\
\hline $\mathbf{5}$ & Tylosin & 20.97 \\
\hline $\mathbf{6}$ & Sulpha drugs & 14.11 \\
\hline
\end{tabular}

(Numerical figures indicate \%)

\section{Mass media exposure}

Majority of the small farmers $(64.52 \%)$, medium farmers $(62.60 \%)$ and large farmers $(62.54 \%)$ had medium level of exposure to the mass media in their respective categories (Table 12).

Among small farmers, $18.56 \%$ belonged to high mass media exposure and rest small farmers had low mass media exposure. Similarly $14.4 \%$ of medium farmers and $24.09 \%$ of large farmers had high level of mass media exposure in their respective categories.

\section{Extension contact}

Majority of small holder dairy farmers (74.40\%) had medium level of extension contact followed by $17.26 \%$ with low level of extension contact and rests of the farmers were having high extension contact. Similarly majority of the medium farmers were having medium level of extension contact followed by $41.67 \%$ of the farmers with low level of extension contact and remaining (7.74\%) were having high extension contact (Table 13). Among large farmers majority $(66.07 \%)$ revealed same pattern with medium extension contact followed by $24.40 \%$ of farmers with low extension contact and rest with high level of extension contact. It was found that large farmers were having high level of extension contact as compared to the small farmers.

\section{Factors affecting "over the counter"} purchase of antibiotics

Large farmers never prioritized the price as the alternative to sought judicious use of antibiotics as evident in the table. Small farmers $(75 \%)$ preferred the low cost antibiotics to treat the bacterial diseases in the herd.

Previous experience gives the insight in to use residual antibiotics which were not considered by large farmers $(75.00 \%)$, while small farmers $(51.79 \%)$ frequently looked for this 
option (Table 14). Ease of obtaining the antibiotics were considered by small farmers $(57.15 \%)$, while large farmers $(66.07 \%)$ never compromised with the effectiveness of treatment by considering the ease of availability.

Practices performed while use of
antibiotics

The decision regarding the best practices and judicious use of antibiotics were acknowledged by large farmers $(72.75 \%)$ on the basis of consistency in decision making (Table 15). Large farmers $(68.78 \%)$ practiced the recording of the use of antibiotics frequently where as small farmers were not aware of this practice. Production goals in relation to preventive use of antibiotics were considered by large farmers $(48.94 \%)$ due to their large herd size. The veterinary consultancy was affected by the financial condition of the farmers, in which large farmers $(85 \%)$ were having the relative advantage.

\section{Practices attributing to antibiotic resistance}

Discriminating the cattle and buffalo for antibiotics treatment by considering the lactation stage aimed to minimize economy of treatment were practiced mostly by small farmers $(56.85 \%)$ frequently. Large farmers $(83.32 \%)$ gave the recommended doses of antibiotic treatment without any alteration while small farmers changed the formulation and doses frequently as per convenience (Table 16). The sale of milk of cow treated with antibiotics were prevalent among all the small, medium and large farmers without a significant difference recognised with due consideration to the economy of production. The uses of left over antibiotics were prevalent among small farmers $(78.42 \%)$ as the regular practice by them for its contingent use.

\section{Resistance to antibiotics prevalent in the study area}

Maximum resistance were observed for Escherichia coli, Pseudomonas aeruoginosa, Streptococcus, Staphylococcus aureus and Salmonella by the veterinarians. Penicillin, Gentamycin, Tetracycline, Tyrosine and sulfa-drugs were found having maximum resistance (Table 17).

\section{Education}

Education broadens the mental horizon at the same time it increases the readiness and predisposition to the acquisition of the new and novel ideas. Increased in level of knowledge was pointedly associated with the use of antibiotics for preventative purpose, antibiotics purchase from medical-stores, complications experienced in animals after having giving antibiotics and the educational level of the farmer (Redding et al., 2014). The educational campaigns can be relatively successful to increase awareness about the resistance issue across the world (Harbarth et al., 2015). Reinforcing the education and fostering the excellence among veterinarians about antibiotic conservation practices have a crucial role to play in containing antibiotic resistance from farm-to-fork (OIE, 2016)

\section{Herd size}

Use of antibiotics in dry cow therapy to cure mastitis occurrences, had very low effects on resistance among bacteria and minimal inhibitory concentration (MIC) of Escherichia coli in herd as well as its environment (Rollins et al.,1974).The good alternatives to above problems can be Organic dairy herds which receive minimal or no antibiotics drugs. More healthy conditions were present in organic than conventional herds (Pol and Ruegg, 2007). 


\section{Annual Income}

The large farmers have judicious use of antibiotics, however farmers with higher incomes had pointedly higher levels of education and as result may adopt better management practices to improve the general health of their animals at their farm (Redding et al., 2014).

The diffusion of dairy farming practices involving the judicious use of antibiotics is lacking in India due to lack of awareness and surveillance programmes. Large farmers were having better financial conditions and herd size. They were innovative and educated too, so the percolations of efforts to diffuse the prudent use of antibiotics were addressed to them in the target segmentation strategy. Small farmers though need more frequent insight to address the issue of antibiotic resistance but they were having low extension contact and mass media exposure. They were practicing the animal husbandry as per convenience and economic consideration so designing the policies and awareness programmes are necessary at their level of understanding and practices.

\section{Further research}

In future, research can be conducted in determining the policies and synthesis of strategies to address the small holder dairy farmers regarding the judicious use of antibiotics. The burden of antibiotic resistance is increased in the world and aggravated by low income and developing countries. So the co-ordination in policy formulation and its implementation should comply with international standards and mandates as mediated by WHO and FAO.

\section{Acknowledgement}

Author(s) acknowledge the support of ICAR-
National Dairy Research Institute, Karnal for providing the Institute fellowship during the research work conducted in partial fulfilment for award of master degree in extension education.

\section{References}

Cecchini, M., Langer, J. and Slawomirski, L., 2015. Antimicrobial Resistance in G7 Countries and Beyond: Economic Issues, Policies and Options for Action. Paris: Organization for Economic Co-operation and Development.

Ceri, H., Olson, M.E. and Turner, R.J., 2010.Needed, new paradigms in antibiotic development. Expert opinion on pharmacotherapy, 11(8), 1233-1237.

Falentin, H., Rault, L., Nicolas, A., Bouchard, D.S., Lassalas, J., Lamberton, P., Aubry, J.M., Marnet, P.G., Le Loir, Y. and Even, S., 2016. Bovine teat microbiome analysis revealed reduced alpha diversity and significant changes in taxonomic profiles in quarters with a history of mastitis. Frontiers in microbiology, 7.

FDA - Food and Drug Administration, 2010.CVM Updates - CVM Reports on Antimicrobials Sold or Distributed for Food-Producing Animals, MD: Food and Drug Administration, Silver Spring.

Founou, L.L., Founou, R.C. and Essack, S.Y., 2016. Antibiotic resistance in the food chain: a developing countryperspective. Frontiers in microbiology, 7.

Harbarth, S., Balkhy, H.H., Goossens, H., Jarlier, V., Kluytmans, J., Laxminarayan, R., Saam, M., Van Belkum, A. and Pittet, D., 2015. Antimicrobial resistance: one world, one fight! Antimicrobial Resistance and Infection Control, 4(1), 49.

McKenna, M., 2013. Antibiotic resistance: the last resort, Nature, Vol. 499(7459), 394- 
396.

Pol, M. and Ruegg, P.L., 2007.Relationship between antimicrobial drug usage and antimicrobial susceptibility of grampositive mastitis pathogens. Journal of dairy science, 90(1), 262-273.

Redding, L.E., Cubas-Delgado, F., Sammel, M.D., Smith, G., Galligan, D.T., Levy, M.Z. and Hennessy, S., 2014. The use of antibiotics on small dairy farms in rural Peru. Preventive veterinary medicine, 113(1), 88-95.

Robinson, T.P., Bu, D.P., Carrique-Mas, J., Fèvre, E.M., Gilbert, M., Grace, D., Hay, S.I., Jiwakanon, J., Kakkar, M., Kariuki, S. and Laxminarayan, R., 2016. Antibiotic resistance is the quintessential One Health issue. Transactions of the Royal Society of Tropical Medicine and Hygiene, 110(7), 377-380.

Rollins, L.D., Pocurull, D.W., Mercer, H.D., Natzke, R.P. and Postle, D.S., 1974. Use of antibiotics in a dairy herd and their effect on resistance determinants in enteric and environmental Escherichia coli. Journal of dairy science, 57(8), 944-950.

Sommer, M.O., Munck, C., Toft-Kehler, R.V. and Andersson, D.I., 2017. Prediction of antibiotic resistance: time for a new preclinical paradigm? Nature Reviews Microbiology, nrmicro-2017.

Van Boeckel, T.P., Brower, C., Gilbert, M., Grenfell, B.T., Levin, S.A., Robinson, T.P., Teillant, A. and Laxminarayan, R., 2015. Global trends in antimicrobial use in food animals. Proceedings of the National Academy of Sciences, 112(18), 5649-5654.

World Health Organization, 2015. World health statistics 2015.World Health Organization.

World Organization for Animal Health (OIE), 2016.Terrestrial Animal Health Code. Paris.

\section{How to cite this article:}

Vikash Kumar and Jancy Gupta. 2018. Socioeconomic Factors and Use of Antibiotics by Dairy Farmers vis-a-vis Antibiotic Resistance. Int.J.Curr.Microbiol.App.Sci. 7(1): 3533-3547. doi: https://doi.org/10.20546/ijcmas.2018.701.415 